\title{
Impacto psicológico do diagnóstico do câncer de mama: um estudo a partir dos relatos de pacientes em um grupo de apoio
}

\author{
Natália Nogueira Teixeira de Menezes \\ Vera Lucia Schulz. \\ Rodrigo Sanches Peres \\ Universidade Federal de Uberlândia
}

\begin{abstract}
Resumo
Este estudo objetivou analisar o impacto psicológico do diagnóstico do câncer de mama a partir dos relatos apresentados espontaneamente por mulheres acometidas pela doença durante as sessões de um grupo de apoio. Utilizando-se o critério de saturação, foram selecionadas 18 sessões do referido grupo de apoio, das quais, no total, participaram 93 mulheres. Os dados coletados foram submetidos a uma apreciação qualitativa a partir do empreendimento de uma análise temática de conteúdo. Os resultados obtidos apontam que o diagnóstico do câncer de mama causou, entre uma parcela expressiva das participantes, um impacto psicológico importante, uma vez que desencadeou experiências de surpresa e tensão, ensejou demonstrações de aceitação e força, motivou tentativas de explicação, redefiniu relacionamentos e intensificou o recurso à religiosidade. $\mathrm{O}$ delineamento de cada um desses processos, em suas dimensões positivas e negativas, fornece elementos profícuos para o aperfeiçoamento da assistência psicológica oferecida a tal população.
\end{abstract}

Palavras-chave: psico-oncologia; neoplasias mamárias; processos grupais.

\begin{abstract}
Breast cancer diagnosis' psychological impact: a study since patients' reports in a support group. This study aimed to analyze breast cancer diagnosis' psychological impact since reports spontaneously presented during sessions of a support group for women diagnosed with this disease. Using the saturation criterion, 18 sessions of the referred support group were selected, in which, in total, 93 women participated. The collected data were submitted to a qualitative appreciation by a thematic analysis of content. The obtained results show that breast cancer diagnosis caused, among an expressive number of participants, an important psychological impact, once unchained surprise and tension experiences, stimulated acceptance and force demonstrations, motivated explanation attempts, redefined relationships and intensified religiosity. The comprehension of each one of those processes, in their positive and negative dimensions, supplies useful elements for the improvement of psychological services offered to this population.
\end{abstract}

Keywords: psycho-oncology; breast cancer, group processes.

\section{Introdução}

O câncer de mama é considerado o tipo de neoplasia maligna mais frequente na população feminina, posto que, conforme as estimativas mais atuais da International Agency for Research on Cancer, cerca de 1.380.000 mulheres foram diagnosticadas no mundo em 2008, totalizando $23 \%$ dos casos de todos os tipos de câncer (Ferlay et al., 2010). No país, as projeções do Ministério da Saúde apontam que, somente no território nacional, aproximadamente 52.000 novos casos serão registrados em 2012 e que o risco variará de 69 casos para cada 100.000 mulheres na região Sudeste a 32 casos para cada 100.000 mulheres na região Nordeste (Brasil, 2011). As taxas de incidência têm se mantido elevadas nos últimos anos, o que pode ser entendido, basicamente, como reflexo da tendência global à predominância de estilos de vida que fomentam a exposição a fatores de risco.
Diversos autores - Rossi e Santos (2003), Maluf, Mori e Barros (2005) e Pelegrini, Cerqueira e Peres (2008), dentre outros - afirmam que o câncer de mama incide sobre o principal símbolo corpóreo da feminilidade, da sensualidade, da sexualidade e da maternidade, de modo que compromete não somente a condição física da paciente, mas também sua saúde mental. Por essa razão, a comunidade científica vem dedicando grande atenção aos aspectos psicológicos da doença há, pelo menos, duas décadas. As pesquisas sobre o assunto podem, de forma geral, ser situadas no campo da Psico-Oncologia, especialidade de caráter multidisciplinar definida por Holland (1990) como a área de interface entre a Psicologia e a Oncologia voltada à exploração das dimensões emocionais do câncer.

Segundo Carvalho (2002), a Psico-Oncologia surgiu como um desdobramento da considerável redução dos índices 
de mortalidade da maioria dos tipos de câncer observada em diversos países, a qual, por sua vez resultou dos avanços técnicocientíficos que tornaram os tratamentos mais resolutivos. Além disso, vale destacar que a Psico-Oncologia se alinha tanto aos objetivos fundamentais da prática profissional quanto aos tópicos básicos da investigação científica em Psicologia da Saúde. Sendo assim, como bem observa Costa Júnior (2001), o principal desafio que se apresenta a esta também se aplica àquela: a estruturação de um modelo de atuação inspirado na concepção biopsicossocial capaz de contribuir efetivamente para a promoção e a manutenção da saúde. A superação desse desafio demanda, para o referido autor, uma maior articulação entre a pesquisa e a assistência.

Schulz, Menezes e Peres (2010) empreenderam uma revisão sistemática da produção científica nacional em Psico-Oncologia, contemplando mais especificamente as pesquisas empíricas dedicadas aos aspectos psicológicos do câncer de mama publicadas em periódicos científicos disponíveis na base de dados SciELO-Brasil. Os autores observaram que tais pesquisas, em sua maioria, exploram as repercussões emocionais do tratamento. Esse enfoque se justifica tendo em vista que as modalidades terapêuticas usualmente preconizadas para o controle do câncer de mama - cirurgia, quimioterapia e radioterapia - promovem consequências físicas que, segundo Müller, Hoffman e Fleck (2006), afetam a mulher em diversos níveis, ensejando, não raro, uma ruptura com a identidade feminina. Tal processo, ressaltese, traz desdobramentos relacionais importantes, posto que é capaz de promover uma ressignificação - em um movimento de continuidade e, ao mesmo tempo, negação da sua caracterização anatômica - dos papéis associados culturalmente à mulher (Aureliano, 2009).

No entanto, em um momento que precede a implementação de qualquer tipo de tratamento, a confirmação do diagnóstico do câncer de mama, por si só, pode desencadear um sofrimento psicológico acentuado à mulher, o qual, vale enfatizar, tende a afetar seu universo de relações, levando-a a se aproximar ou se afastar daqueles que a cercam. Sabe-se que uma parcela significativa dos pacientes oncológicos que há algumas décadas estaria condenada consegue, nos dias de hoje, sobreviver à doença e, ainda que com algumas restrições, dar continuidade à sua vida. A despeito disso, o câncer, como bem observa Carvalho (2002), permaneceu sendo visto por muitas pessoas como uma inapelável sentença de morte, representando popularmente, assim, o mais cruel dos vaticínios. E o câncer de mama, mais especificamente, se destaca como a doença mais temida pelas mulheres (Venâncio, 2004). Torna-se patente, diante do exposto, que se faz necessário dedicar atenção especial ao impacto emocional de seu diagnóstico.

De acordo com a revisão sistemática de Schulz et al. (2010), porém, poucas pesquisas nacionais têm sido desenvolvidas recentemente com esse tema. Constituem exceções nesse sentido os estudos de Peres e Santos (2007), Ferreira, Almeida e Rasera (2008) e Scorsolini-Comim, Santos e Souza (2009). O primeiro investiga as reações emocionais à doença e suas implicações em termos do ajustamento psicossocial das pacientes. Já o segundo se dedica aos sentidos atribuídos ao diagnóstico por pacientes e seus parceiros. O terceiro, por fim, contempla as transações subjetivas estabelecidas pelas pacientes com o ambiente no manejo dos desdobramentos sociais e afetivos tanto do diagnóstico quanto do tratamento.

Deve-se mencionar que o impacto psicológico do diagnóstico do câncer de mama é um tema recorrente na literatura científica internacional. Os estudos de Payne, Sullivan e Massie (1996), McBride, Clipp, Peterson, Lipkus e Demark (2000) e Grassi (2005) o atestam. Porém, os resultados oriundos de pesquisas sobre o assunto realizadas em outros países muitas vezes não podem ser devidamente generalizados para o contexto nacional, pois se referem a pacientes que, de forma geral, dispõem de um sistema de saúde pública que, privilegiando medidas de prevenção secundária, viabiliza a detecção precoce da doença. Consequentemente, o tratamento, em contraste com o que se observa tipicamente no Brasil, envolve o emprego de recursos mais conservadores e atinge maior resolutividade, restringindo as chances de recidiva e aumentando a sobrevida da paciente. Nesse cenário, o impacto psicológico do diagnóstico tende, em boa parte dos casos, a ser atenuado.

É preciso enfatizar também que, como observa Löwy (2010), em países como os Estados Unidos da América, as atitudes em relação ao câncer de mama, tanto dos profissionais de saúde quanto da população em geral, diferem daquelas que se observam no contexto brasileiro. Para a referida autora, isso ocorre basicamente porque, nesses países, as noções de "risco" e "prevenção" são levadas ao extremo em função de determinantes culturais. Como resultado, é frequente a utilização supostamente profilática de cirurgias agressivas como a mastectomia bilateral - que consiste na extirpação total das duas mamas - em pessoas assintomáticas nas quais alguma mutação associada à doença é identificada a partir de rastreamento genético. Segundo Romeira (2007), esse tipo de experiência tem implicações específicas para a mulher, sobretudo em termos relacionais, uma vez que pode colocar em relevo uma problemática associada à matriz familiar.

Outra ressalva que se aplica à generalização dos resultados oriundos de pesquisas sobre o impacto psicológico do diagnóstico do câncer de mama desenvolvidas em outros países se refere ao fato de algumas delas defenderem a existência de um conjunto de respostas emocionais que se organizam em fases, o que enseja um reducionismo da complexidade inerente ao assunto e se revela pouco proveitoso na prática clínica. De qualquer forma, revisões sistemáticas desenvolvidas por Owen, Klapow, Hicken e Tucker (2001) e Edwards, Hailey e Maxwell (2008) comprovam que a assistência psicológica, em diferentes culturas, pode auxiliar mulheres acometidas por câncer de mama a adotar estratégias de enfrentamento adaptativas face ao diagnóstico, o que viabiliza uma melhor adesão ao tratamento. Ademais, é igualmente capaz de potencializar a tolerância aos efeitos colaterais da cirurgia, da radioterapia e da quimioterapia, minimizando o incômodo desencadeado por eventuais dores, edemas, náuseas e infecções e diminuindo tanto a frequência quanto a intensidade de intercorrências.

A assistência psicológica a mulheres acometidas por câncer de mama pode ser executada mediante o emprego dos mais diversificados recursos técnicos. Não obstante, as práticas grupais, para diversos autores - Holland e Holahan (2003), Pinheiro, Silva, Mamede e Fernandes (2008) e Santos, Prado, 
Panobianco e Almeida (2011), dentre outros - são especialmente proveitosas, pois a universalidade da queixa facilita a expressão de sentimentos e a troca de experiências relacionadas à doença e a seu tratamento, o que tende a diminuir o nível de ansiedade das participantes e a potencializar a ocorrência de processos de mudança. Ademais, sentimentos de isolamento e solidão podem ser trabalhados em grupo, o que auxilia a diminuir o estigma associado ao câncer de mama (Scorsolini-Comim et al., 2009).

Tendo em vista o que precede, foi instituído um estágio curricular profissionalizante para alunos do curso de graduação em Psicologia de uma universidade pública, por meio do qual tem sido oferecido um grupo de apoio a mulheres acometidas por câncer de mama vinculadas ao ambulatório especializado de um hospital universitário. São atendidas nesse ambulatório pacientes que se encontram em diferentes momentos, sendo que algumas passam por consultas médicas subsequentes à confirmação do diagnóstico para a definição dos tratamentos a serem realizados, ao passo que outras, em fase de reabilitação, apenas realizam seguimento para detecção precoce de uma eventual recidiva e prevenção de complicações tardias.

Em seu atual enquadre, vigente desde Fevereiro de 2009, o grupo de apoio em questão tem ocorrido com periodicidade semanal. Em que pese o fato de se tratar de uma modalidade assistencial de duração ilimitada, visto que sua oferta tem sido contínua ao longo do tempo, a extensão de cada uma de suas sessões é circunscrita, totalizando, em média, uma hora, com o intuito de não implicar em atrasos para as consultas médicas das participantes. É válido esclarecer ainda que as intervenções verbais executadas pelo coordenador no manejo técnico do grupo dependem do conteúdo abordado, mas, de modo geral, predominam intervenções suportivas como o reasseguramento, a sugestão e a validação.

A experiência com o referido grupo de apoio, embora bem-sucedida, aponta que se faz necessária uma compreensão mais aprofundada do impacto psicológico do diagnóstico do câncer de mama para que se possa aprimorar a assistência oferecida às mulheres que se encontram na etapa inicial de uma longa batalha a ser travada com a doença. O presente estudo foi concebido nesse sentido, propondo-se a articular ensino, pesquisa e extensão, uma vez que visa à produção e difusão do conhecimento a partir da experiência em uma modalidade assistencial oferecida à população como parte das atividades de um estágio curricular profissionalizante. Desse modo, justificase por subsidiar uma relação dialética entre teoria e prática e, concomitantemente, auxiliar a estreitar os laços da universidade com a comunidade.

Assumindo a premissa de Scorsolini-Comim et al. (2009), segundo a qual o contexto grupal é especialmente favorável para a discussão de experiências relacionadas à nova realidade implementada pela descoberta da doença, optou-se por privilegiá-lo como locus para a coleta de dados. Afinal, parece razoável propor que a exploração dos relatos apresentados espontaneamente por mulheres acometidas por câncer de mama acerca do impacto psicológico da doença pode contribuir para o avanço do conhecimento que atualmente se tem a respeito do assunto. $O$ presente estudo, portanto, teve como objetivo analisar o impacto psicológico do diagnóstico do câncer de mama a partir dos relatos apresentados espontaneamente por mulheres acometidas pela doença durante as sessões de um grupo de apoio.

\section{Método}

\section{Composição do corpus}

O corpus do presente estudo foi composto por 18 sessões do referido grupo de apoio. No total, 93 pacientes estiveram presentes nessas 18 sessões. Vale destacar que se trata de um grupo de ocorrência única, ou seja, a cada sessão sua composição é diferente, já que dele participam as pacientes com consulta médica agendada para o dia no ambulatório ao qual as mesmas se encontram vinculadas. Algumas dessas pacientes estiveram presentes em diversas sessões, ao passo que outras em apenas uma. Ressalte-se que a periodicidade das consultas médicas varia muito, sendo mensal em alguns casos ou anual em outros. A isso, portanto, se deve o fato de o número total de pacientes ser relativamente elevado tendo em vista o número total de sessões consideradas para os fins do presente estudo.

Deve-se esclarecer que, de modo geral, são atendidas no ambulatório em pauta mulheres de baixa renda, casadas ou amasiadas, com baixo nível de escolaridade, faixa etária entre 50 e 70 anos e oriundas tanto da cidade na qual se situa o hospital universitário quanto de suas cercanias. Por fim, é preciso salientar que a definição do número de sessões selecionadas para a composição do corpus do presente estudo foi executada com base no critério de saturação, o qual, a propósito, tem sido amplamente utilizado no campo das pesquisas qualitativas. Portanto, como preconizam Fontanella, Ricas e Turato (2008), envolveu a identificação, a partir da avaliação dos pesquisadores, de certa redundância nos relatos referentes ao impacto psicológico do diagnóstico do câncer de mama apresentados pelas pacientes.

\section{Coleta de dados}

A coleta de dados do presente estudo foi realizada junto aos relatórios das sessões do grupo de apoio em questão. Tais relatórios foram elaborados a partir de anotações que reproduzem o mais fielmente possível os relatos apresentados pelo coordenador e pelas participantes do início ao final de cada uma das sessões. As anotações foram executadas durante as sessões por dois observadores silentes devidamente treinados para tanto, e posteriormente organizadas pelos mesmos em relatórios únicos, sendo um por sessão, os quais respeitam a sequência dos acontecimentos. Ressalte-se que todos os relatórios foram apreciados em supervisão e arquivados como documentos.

\section{Desenho metodológico}

Diante do exposto, conclui-se que o desenho metodológico documental foi privilegiado no presente estudo. Trata-se de um desenho metodológico de grande relevância em Ciências Humanas, de acordo com o qual documentos de diversos tipos são adotados como fonte de informação e, para tanto, devem ser analisados em função da finalidade da pesquisa (Pimentel, 2001). Partindo da categorização proposta por Bauer, Gaskell e Allum (2002/2000), os relatórios podem ser considerados documentos formais por necessitarem de treinamentos e conhecimentos 
especializados para sua produção. Porém, os relatórios também se assemelham ao que os referidos autores designam como documentos informais. $\mathrm{O}$ interesse pelo modo como as pessoas se expressam norteia a elaboração dos documentos desse tipo, mas a influência da visão do pesquisador não deve ser desconsiderada. Nesse sentido, os relatórios foram analisados no presente estudo como um meio-termo entre os documentos formais e os documentos informais.

Vale destacar que se optou por utilizar relatórios como fonte de informações com o intuito de assegurar que os relatos das pacientes seriam representativos dos conteúdos veiculados espontaneamente no grupo de apoio da maneira que o mesmo vem sendo realizado rotineiramente, ou seja, sem criar uma situação especial para a coleta de dados. O presente estudo, portanto, se alinha ao enfoque naturalístico, partindo-se do princípio de que não teve como intuito principal testar hipóteses previamente formuladas, mas, sim, descrever um fenômeno assim como ele naturalmente ocorre e nas circunstâncias que espontaneamente o geram (Selltiz, Jahoda, Deutsch, \& Cook, 1972/1965)

Considerando-se os objetivos propostos, optou-se por privilegiar análises descritivas e exploratórias a partir de um enfoque qualitativo. Conforme Minayo e Sanches (1993, p.247), uma pesquisa qualitativa aborda basicamente valores, crenças, representações, hábitos, atitudes e opiniões e se mostra especialmente proveitosa "para aprofundar a complexidade de fenômenos, fatos e processos particulares e específicos de grupos mais ou menos delimitados em extensão e capazes de serem abrangidos intensamente". Busca-se, basicamente, interpretar fenômenos nos termos das significações que as pessoas atribuem a eles (Denzin \& Lincoln, 2000). Portanto, uma pesquisa qualitativa, para Camic, Rhodes e Yardley (2003), é particularmente oportuna para a abordagem de experiências humanas complexas, das quais o adoecimento por câncer de mama pode ser considerado um exemplo.

\section{Análise de dados}

Optou-se por utilizar no presente estudo a análise temática de conteúdo proposta por Bardin (1979/1970), a qual se destaca como uma das principais técnicas de análise de dados no âmbito das pesquisas qualitativas. Trata-se, conforme Minayo, (1992), de uma ferramenta metodológica capaz de subsidiar a identificação de significados latentes imperceptíveis no conteúdo manifesto de uma mensagem a partir do desenvolvimento de inferências e deduções. Desse modo, a análise temática de conteúdo preconiza que a característica polissêmica inerente à comunicação humana emerge quando do empreendimento de operações lógicas que envolvem a codificação de elementos discursivos em classes de equivalência (Bardin, 1979/1970). Vale destacar que foram submetidos à análise temática de conteúdo, mais especificamente, os relatos referentes ao impacto psicológico do diagnóstico do câncer de mama, de modo que os relatos referentes a outros assuntos não foram considerados para os fins do presente estudo.

Assim, os relatos selecionados foram analisados mediante a execução dos três procedimentos básicos que constituem a análise temática de conteúdo, a saber: pré-análise, exploração do material e tratamento dos resultados. O primeiro demanda um contato exaustivo com o conjunto do material por meio de leituras flutuantes, sua organização em temas - ou seja, em unidades de significação naturalmente circunscritas - e o delineamento de hipóteses iniciais. Já o segundo consiste essencialmente na administração de técnicas voltadas à codificação do conteúdo e à agregação dos temas. O terceiro tem como etapa inicial a seleção dos resultados decorrentes da exploração do material e como etapa complementar a elaboração de inferências, interpretações e sínteses (Bardin, 1979/1970). Ressalte-se que, para que cada um dos referidos procedimentos possa ser bem-sucedido, há que se articular o conjunto do material com o contexto no qual o mesmo foi produzido e os múltiplos fatores que podem exercer influência nesse processo.

\section{Aspectos éticos}

O presente estudo foi submetido a um Comitê de Ética em Pesquisa, em conformidade com a Resolução 196/96 do Conselho Nacional de Saúde, tendo obtido parecer favorável. Portanto, todos os aspectos éticos pertinentes foram observados em seu desenvolvimento. Nesse sentido, tomou-se o cuidado, ao longo do texto, de substituir o nome verdadeiro das pacientes por nomes fictícios quando da apresentação de seus relatos sobre o impacto psicológico do câncer de mama. Buscou-se, assim, preservar-lhes o anonimato, o que evidencia que o respeito às mesmas foi uma preocupação central do presente estudo.

\section{Resultados e discussão}

Das 93 pacientes que participaram das 18 sessões consideradas para os fins do presente estudo, 46 veicularam espontaneamente, no total, 79 relatos referentes ao impacto psicológico do câncer de mama. Como se vê na Tabela 1, tais relatos foram agregados, em função das unidades de sentido que puderam ser identificadas a partir da análise de conteúdo, em 7 temas principais, a saber: a) surpresa e tensão, b) aceitação e força, c) tentativas de explicação, d) religiosidade, e) relacionamentos, f) atendimento da equipe médica e g) alterações emocionais permanentes. Para os fins do presente estudo, contudo, serão privilegiados, devido a limitações de espaço, os 5 primeiros temas.

Vale destacar que o fato de uma parcela das pacientes que participaram das sessões consideradas para os fins do presente estudo não ter veiculado relatos sobre o assunto ora abordado era esperado. Afinal, como já mencionado, frequentam o grupo tanto mulheres recém-diagnosticadas, que se encontram na fase de definição dos tratamentos a serem realizados, quanto mulheres em reabilitação, já em remissão da doença, as quais apenas realizam seguimento de rotina. Logo, seria de se imaginar que, assim como de fato ocorreu, referências sobre o impacto psicológico do diagnóstico fossem mais comuns nos relatos das primeiras do que nos relatos das segundas.

\section{Surpresa e tensão}

Ao receberem o diagnóstico do câncer de mama, algumas pacientes se revelaram totalmente surpresas, como se vê no relato 1, o que pode ensejar experiências de tensão acentuada: "Esse 
Tabela 1

Distribuição das participantes de acordo com as unidades de sentido que configuraram os temas referentes ao impacto psicológico do câncer de mama

\begin{tabular}{lll}
\hline \multicolumn{1}{c}{ Categorias } & \multicolumn{1}{c}{ Participantes } & Frequência \\
\hline Surpresa e tensão & $5,13,22,29,35,38,39,51,52,53,54,55,56$, & 19 \\
& $57,77,83,89,90$ e 93 & 18 \\
Aceitação e força & $2,5,9,19,27,31,35,38,44,55,59,61,69,83$, & 12 \\
Tentativas de explicação & $89,90,91$ e 92 & 13 \\
$\begin{array}{l}\text { Religiosidade } \\
\text { Relacionamentos }\end{array}$ & $2,4,5,19,35,38,40,48,53,67,82$ e 83 & 9 \\
$\begin{array}{l}\text { Atendimento da equipe } \\
\text { médica }\end{array}$ & $36,38,44,51,60,61,81,83$ e 93 & 5 \\
Alterações emocionais & $9,12,56,68$ e 71 & 5 \\
permanentes & 61,66 e 92 & 3 \\
\hline
\end{tabular}

negócio de câncer, a gente [...] nunca pensa que vai acontecer com a gente" (Alice, paciente 5). É possível propor que isso ocorreu porque a confirmação da doença as colocou em contato com a finitude inerente à vida, uma vez que o câncer evidencia a fragilidade da condição humana. Afinal, o câncer, conforme Chiattone (1996), mostra ao portador que seu mundo anterior não existe mais, que sua existência está ameaçada e que o amanhã não se estenderá para sempre. Ademais, deve-se destacar que a morte ainda não é encarada como um fenômeno natural na cultura ocidental, mas sim como um acontecimento temível, um incidente a ser evitado a todo custo (Franco, 2002).

Parte das pacientes que se revelaram surpresas e tensas quando da confirmação do diagnóstico relataram que não sabiam exatamente o que fazer frente a essa situação, já que experimentaram uma desestruturação pessoal e familiar significativa. Ocorre que, aparentemente, algumas mulheres acometidas por doenças graves como o câncer se vêem totalmente à mercê do destino, de modo que encontram dificuldade para tomar decisões apropriadas em relação ao tratamento. Refletindo tal dificuldade, essas mulheres anseiam para que o tempo passe mais rápido e, consequentemente, o tratamento logo se conclua, como se vê no relato 2: "Eu tenho medo também. Mas acima de tudo ansiedade pra terminar isso..." (Berenice, paciente 39). A dificuldade de conviver com a tensão instalada pelo diagnóstico da doença pode, ainda, provocar como desdobramento a superestimação da terapêutica cirúrgica, fenômeno que se baseia na crença de que a remoção do tumor será suficiente para garantir o desaparecimento da enfermidade (Maluf et al., 2005). O relato 3 exemplifica esse fenômeno: “... Vou pegar [o tumor], jogar fora e pronto" (Carolina, paciente 54).

\section{Aceitação e força}

Verificou-se que, quando do diagnóstico, parte das pacientes deu início a um processo lento e gradual de aceitação da doença. As mesmas apresentaram, na etapa inicial desse processo, atitudes de enfrentamento que variaram de uma aceitação mais passiva, com elementos de conformismo, a uma aceitação mais ativa, reveladora, sobretudo mediante a busca de informações, de uma postura de contraposição em relação à doença com espírito de luta. Ilustram esse achado, respectivamente, os relatos $4 \mathrm{e}$ 5: "Antes tirar [o tumor] do que a gente ficar prejudicada toda a vida" (Isabela, paciente 2) e "Pesquisei na internet todas as fases do câncer" (Daniela, paciente 31). Segundo Peres e Santos (2007), o conformismo pode contribuir para a redução temporária do estresse da paciente acometida por câncer de mama, porém, tende a dificultar o ajustamento psicossocial a longo prazo. Já o espírito de luta se mostra mais apropriado, na medida em que fomenta a superação das dificuldades inerentes ao tratamento e diminui o estigma associado ao diagnóstico.

Vale destacar o relato de que algumas das pacientes que se mostraram conformistas em relação à doença procuravam se afastar de sentimentos supostamente negativos como o medo e a tristeza. Segundo Piro, Zeldow, Knight, Mytko e Gradishar (2001), entretanto, há uma correlação positiva entre a inibição emocional quando do diagnóstico e a ocorrência de transtornos do humor e déficits no desempenho funcional ao longo do tratamento em mulheres acometidas por câncer de mama. Logo, deve-se dedicar atenção especial às pacientes que apresentam esse tipo de reação.

A paciente que apresentou o relato 6 sugere, inclusive, que entendia que, sentindo-se triste, estaria prejudicando sua própria recuperação: "A tristeza só atrapalha [o tratamento], por isso eu dou "xô" para a tristeza" (Fabiana, paciente 9). Já a paciente que apresentou o relato 7 sustenta que, contrariando o que seria esperado, jamais temeu o câncer: "Em nenhum momento tive medo da doença" (Laura, paciente 44). Mas o relato 8 parece mais verossímil, na medida em que a paciente que o apresentou denota que, a despeito de ter acreditado que seria capaz de não se deixar abater emocionalmente em função do diagnóstico, reconheceu a dificuldade inerente a essa empreitada: “A gente pensa que vai ser forte até o final, mas é muito difícil” (Aparecida, paciente 83).

Diante do exposto, constata-se que os relatos 6 e 7 possivelmente foram influenciados por um intrigante fenômeno conhecido como "tirania do pensamento positivo". Tal fenômeno, segundo Holland e Lewis (2000), faz com que o paciente oncológico se sinta pressionado - tanto interna quanto externamente - a experimentar apenas emoções consideradas positivas e, assim, a reprimir sentimentos supostamente negativos, como se disso dependesse sua sobrevivência. Dessa maneira, enseja uma postura estereotipada, a qual frequentemente evoluiu da resignação estóica à culpabilidade, uma vez que o menor revés no tratamento abala sua sustentação. A descrição 
da tirania do pensamento positivo, portanto, torna patente que não devem ser levados ao extremo os resultados oriundos de pesquisas que - a exemplo daquela conduzida por Carver et al. (2005) - salientam que o otimismo se afigura como uma espécie de proteção contra o impacto psicológico do câncer de mama.

\section{Tentativas de explicação}

Aparentemente, parte das pacientes pesquisadas, tão logo recebeu o diagnóstico, engajou-se em um movimento de autoreflexão com o intuito de identificar os fatores associados ao surgimento do câncer de mama. Trata-se de um processo de extrema relevância, visto que representa o ponto de partida para a explicação, a compreensão e a aceitação da doença e, consequentemente, para a definição das estratégias de enfrentamento a serem adotadas (Lavery \& Clarke, 1996). Deve-se destacar que a existência de fatores emocionais supostamente associados ao câncer de mama foi proposta por uma das participantes, como ilustra o relato 9: "Essa doença pega as pessoas nervosas" (Suzana, paciente 4).

Porém, as demais pacientes que se engajaram em tal movimento de auto-reflexão demonstraram, a partir de seus relatos, que encontravam dificuldades para avançar nesse sentido. Como sugere o relato 10, para algumas delas isso possivelmente aconteceu porque o câncer de mama, como já mencionado, representa a doença mais temida pelas mulheres: "Então isso [a doença] assusta demais. Porque você não sabe o porquê, né?" (Cássia, paciente 82 ). Para outras, é mais provável que a dificuldade em compreender o surgimento da doença se encontre relacionada ao estilo de vida adotado pelas mesmas, o qual minimizava a exposição a fatores de risco como o tabagismo e o consumo abusivo de álcool, conforme o relato 11: "Por que comigo? Eu não bebo, não fumo" (Lurdes, paciente 40).

\section{Religiosidade}

Parte das pacientes pesquisadas mencionou que, diante da confirmação do diagnóstico de câncer de mama, recorreram à religiosidade. Para Ferreira et al. (2008), isso ocorre, sobretudo, quando à doença é atribuído um significado de provação. Mas deve-se esclarecer que a religiosidade pode ser empregada de diversas formas no enfrentamento de uma situação adversa. A maioria dessas pacientes, como se vê no relato 12, aparentemente buscava apoio espiritual, mas não deixava de assumir uma postura ativa diante das dificuldades implementadas pelo diagnóstico: "Eu não estou sozinha, eu estou com Deus" (Letícia, paciente 12). Desse modo, as mesmas engajavam-se em uma espécie de co-responsabilidade com Deus, análoga àquela que caracteriza um estilo de enfrentamento religioso que, conforme Panzini e Bandeira (2007), é denominado "colaboração".

Outras dessas pacientes que recorreram à religiosidade aparentemente outorgaram a Deus a responsabilidade pela cura, como se elas não pudessem colaborar com o próprio tratamento. O relato 13 é ilustrativo nesse sentido: "Eu estou conformada, o que Deus fizer, tá feito" (Madalena, paciente 51). A atitude em questão é típica de um estilo de enfrentamento religioso denominado "delegação", o qual é considerado pouco adaptativo (Panzini \& Bandeira, 2007). Vale destacar que Caetano, Gradim e Santos (2009) e Gonçalves, Gíglio e Ferraz (2005), em estudos nacionais recentes, apontam que a religiosidade auxiliou a minimizar o desespero, a angústia e a depressão em mulheres acometidas por câncer de mama. Entretanto, em tais estudos não se especifica quais foram os estilos de enfrentamento religioso predominantes entre as mulheres pesquisadas.

\section{Relacionamentos}

A partir da análise dos relatos das pacientes no grupo, podese notar que as implicações do diagnóstico em termos de seus relacionamentos interpessoais foram basicamente de dois tipos. Algumas delas referiram que optaram por não compartilhar com familiares a descoberta da doença, como revela o relato 14: "Eu não quis incomodar ninguém, por isso que não contei e por isso o nódulo cresceu muito" (Sara, paciente 36). Nesses casos, uma acentuada tendência ao isolamento foi implementada desde as primeiras suspeitas da doença, o que, inclusive, fez com que a realização dos exames que poderiam confirmá-la ou refutá-la fosse postergada, prejudicando, como consequência, a saúde dessas pacientes em função da evolução do tumor devido à passagem do tempo. Possivelmente isso ocorreu porque, como já mencionado, o câncer, para Carvalho (2002), ainda é visto por muitas pessoas como uma inapelável sentença de morte, de modo que vislumbrar a possibilidade da doença tem, por si só, um efeito paralisante.

Por outro lado, a maioria das pacientes mencionou que preferiu compartilhar as experiências relacionadas ao diagnóstico com seus amigos e familiares. O relato 15 exemplifica essa iniciativa: "O meu marido também me deu força na época [...] Antes de eu adoecer, [o relacionamento] não estava essas coisas, mas ele me deu força, me levava, me buscava das consultas. Aí [o relacionamento] melhorou" (Josefa, paciente 81 ). Tais pacientes afirmaram, inclusive, que seus parceiros as auxiliaram muito durante todo o processo, de modo que ocorreram alterações positivas na dinâmica do casal. Esses achados são compatíveis com aqueles reportados por Biffi e Mamede (2004), já que as autoras em questão constataram que uma relação familiar saudável pode contribuir significativamente para a adaptação à nova condição de vida implementada pelo diagnóstico de câncer de mama, criando um ambiente favorável para que a mulher possa enfrentar a doença com maior segurança.

\section{Considerações finais}

O presente estudo revela que o diagnóstico do câncer de mama causou, entre uma parcela expressiva das participantes, um impacto psicológico importante, tendo desencadeado experiências de surpresa e tensão, ensejado demonstrações de aceitação e força, motivado tentativas de explicação, redefinido relacionamentos e intensificado o recurso à religiosidade. $\mathrm{O}$ delineamento de cada um desses processos, em suas dimensões positivas e negativas, fornece elementos profícuos para o aperfeiçoamento da assistência psicológica oferecida a tal população. Além disso, o presente estudo atesta que o contexto grupal representa uma fonte de dados extremamente rica - e ainda pouco explorada em nosso meio - para a compreensão dos aspectos psicológicos do câncer de mama. Tal achado tem implicações práticas para a definição do desenho metodológico 
de novas pesquisas dedicadas ao assunto.

\section{Referências}

Aureliano, W. A. (2009). “... e Deus criou a mulher”: reconstruindo o corpo feminino na experiência do câncer de mama. Estudos Feministas, 17(1), 49-70.

Bardin, L. (1979). Análise de conteúdo. (L. A. Reto \& A. Pinheiro, Trads.). Lisboa: Edições 70. (Obra original publicada em 1970)

Bauer, M. W., Gaskell, G., \& Allum, N. C. (2002). Qualidade, quantidade e interesses do conhecimento: evitando confusões (P. A. Guareschi, Trad.). In M. W. Bauer \& G. Gaskell (Orgs.), Pesquisa qualitativa com texto, imagem e som: um manual prático (pp.17-36). Petrópolis: Vozes. (Obra original publicada em 2000)

Biffi, R. G., \& Mamede, M. V. (2004). Suporte social na reabilitação da mulher mastectomizada: o papel do parceiro sexual. Revista da Escola de Enfermagem da USP, 38(3), 262-269.

Brasil. Ministério da Saúde. Instituto Nacional do Câncer. (2011). Estimativas 2012: incidência de câncer no Brasil. Rio de Janeiro: Ministério da Saúde.

Caetano, E. A., Gradim, C. V. C., \& Santos, L. E. S. (2009). Câncer de mama: reações e enfrentamento ao receber o diagnóstico. Revista de Enfermagem da UERJ, 17(2), 257-261.

Camic, P. M., Rhodes, J. E., \& Yardley, L. (2003). Naming the stars: integrating qualitative methods into psychological research. In P. M. Camic, J. E. Rhodes, \& L. Yardley (Orgs.), Qualitative research in Psychology: expanding perspectives in methodology and design (pp.3-15). Washington: American Psychological Association.

Carvalho, M. M. M. J. (2002). Psico-oncologia: história, características e desafios. Psicologia USP, 13(1), 151-166.

Carver, C. S., Smith, R. G., Antoni, M. H., Petronis, V. M., Weiss, S., \& Derhagopian, R. P. (2005). Optimistic personality and psychosocial wellbeing during treatment predict psychosocial well-being among long-term survivors of breast cancer. Health Psychology, 24(5), 508-516.

Chiattone, H. B. C. (1996). Uma vida para o câncer. In V. A. Angerami-Camon, H. B. C. Chiattone \& E. A. Nicoletti (Orgs.), O doente, a psicologia e o hospital (pp.73-110). São Paulo: Pioneira.

Costa Júnior, A. L. (2001). O desenvolvimento da Psico-oncologia: implicações para a pesquisa e intervenção profissional em saúde. Psicologia: Ciência e Profissão, 21(2), 36-43.

Denzin, N. K., \& Lincoln, Y. S. (2000). Handbook of qualitative research. Thousand Oaks: Sage.

Edwards, A. G. Hailey, S., \& Maxwell, M. (2008). Psychological interventions for women with metastatic breast cancer. Cochrane Database of Systematic Reviews, 16(3), CD004253.

Ferlay, J., Shin, H. R., Bray, F., Forman, D., Mathers, C., \& Parkin, D. M. (2010). GLOBOCAN 2008: cancer incidence and mortality worldwide. Recuperado de http://globocan.iarc.fr.

Ferreira, C. B., Almeida, A. M., \& Rasera, E. F. (2008). Sentidos do diagnóstico por câncer de mama feminino para casais que o vivenciaram. Interface, 12(27), 863-871.

Fontanella, B. J., Ricas, J., \& Turato, E. R. (2008). Amostragem por saturação em pesquisas qualitativas em saúde: contribuições teóricas. Cadernos de Saúde Pública, 24(1), 17-27.

Franco, M. H. P. (2002). Uma mudança no paradigma sobre o enfoque da morte e do luto na contemporaneidade. In M. H. P. Franco (Org.), Estudos avançados sobre o luto (pp. 15-38). Campinas: Livro Pleno.

Gonçalves, M., Giglio, J. S., \& Ferraz, M. P. T. A. (2005). Religiosidade como fator de proteção contra depressão em pacientes com neoplasia mamária. Arquivos Brasileiros de Psiquiatria, Neurologia e Medicina Legal, 99(4), 16-20.

Grassi, L. (2005). Psychopathology and psychiatric disorders among breast cancer patients. Minerva Psichiatrica, 46(2), 105-117.
Holland, J. C. (1990). Historical overview. In J. C. Holland \& J. Rowland (Orgs.). Handbook of psychooncology: psychological care of the patient with cancer (pp. 3-12). Nova Iorque: Oxford University Press.

Holland, J. C., \& Lewis, S. (2000). The human side of cancer: living with hope, coping with uncertainty. Nova Iorque: Harper Collins.

Holland, K. D., \& Holahan, C. K. (2003). The relation of social support and coping to positive adaptation to breast cancer. Psychology and Health, 18(1), 15-29.

Lavery, J. F., \& Clarke, V. A. (1996) Causal attributions, coping strategies and adjustment to breast cancer. Cancer Nursing, 19(1), 20-28.

Löwy, I. (2010). Preventive strikes: women, precancer, and prophylactic surgery. Baltimore: The John Hopkins University Press.

Maluf, M. F. M., Mori, L. J., \& Barros, A. C. S. D. (2005). O impacto psicológico do câncer de mama. Revista Brasileira de Cancerologia, 51(2), 149-154.

McBride, C. M., Clipp, E., Peterson, B. L., Lipkus, I. M., \& Demark, W. W. (2000). Psychological impact of diagnosis and risk reduction among cancer survivors. Psycho-Oncology, 9(5), 418-427.

Minayo, M. C. S. (1992). O desafio do conhecimento: pesquisa qualitativa em saúde. São Paulo: Hucitec.

Minayo, M. C. S., \& Sanches, O. (1993). Quantitativo-qualitativo: oposição ou complementaridade? Cadernos de Saúde Pública, 9(3), 239-262.

Müller, M. C., Hoffmann, F. S., \& Fleck, P. (2006). A vivência do câncer de mama e a imagem corporal na mulher contemporânea. In B. S. G. Werlang \& M. S. Oliveira (Orgs.), Temas em Psicologia Clínica (pp. 203-208). São Paulo: Casa do Psicólogo.

Owen, J., Klapow, J., Hicken, B., \& Tucker, D. (2001). Psychosocial interventions for cancer: review and analysis using a three-tiered outcomes model. PsychoOncology, 10(3), 218-230.

Panzini, R. G., \& Bandeira, D. R. (2007). Coping (enfrentamento) religioso/ espiritual. Revista de Psiquiatria Clínica, 34(1), 126-135.

Payne, D. K., Sullivan, M. D., \& Massie, M. J. (1996). Womens' psychological reactions to breast cancer. Seminars in Oncology, 23(1), 89-97.

Pelegrini, L. G., Cerqueira, J. A., \& Peres, R. S. (2008). Indicadores de qualidade de vida e sintomas de ansiedade, depressão e estresse em mulheres mastectomizadas no período de reabilitação. Revista da Sociedade de Psicologia do Triângulo Mineiro, 12(2), 168-176.

Peres, R. S., \& Santos, M. A. (2007). Breast cancer, poverty and mental health: emotional response to the disease in women from popular classes. Revista Latino-Americana de Enfermagem, 15(número especial), 786-791.

Pimentel, A. (2001). O método da análise documental: seu uso numa pesquisa historiográfica. Cadernos de Pesquisa, 114, 179-195.

Pinheiro, C. P. O., Silva, R. M., Mamede, M. V., \& Fernandes, A. F. C. (2008). Participating in a support group: experience lived by women with breast cancer. Revista Latino-Americana de Enfermagem, 16(4), 733-738.

Piro, M., Zeldow, P. B., Knight, S. J., Mytko, J. J., \& Gradishar, W. J. (2001). The relationship between agentic and communal personality traits and psychosocial adjustment to breast cancer. Journal of Clinical Psychology in Medical Settings, 8(4), 263-271.

Romeira, R. C. P. M. (2007). Algumas implicações psicológicas da testagem genética para câncer de mama/ovário. Psicologia para a América Latina, 9, 0-0. Disponível recuperado de: <http://pepsic.bvsalud.org/scielo. php?script=sci_arttext\&pid=S1870-350X2007000100012\&lng=pt\&nrm= iso $>$. ISSN $1870-350 \mathrm{X}$.

Rossi, L., \& Santos, M. A. (2003). Repercussões psicológicas do adoecimento e do tratamento em mulheres acometidas pelo câncer de mama. Psicologia: Ciência e Profissão, 23(4), 32-41

Santos, M. A., Prado, M. A. S., Panobianco, M. S., \& Almeida, A. M. (2011) Grupo de apoio a mulheres mastectomizadas: cuidando das dimensões subjetivas do adoecer. Revista da SPAGESP, 12(2), 27-33.

Schulz, V. L., Menezes, N. N. T., \& Peres, R. S. (2010). Pesquisa em psicooncologia no Brasil: desenvolvimentos recentes sobre aspectos psicológicos do câncer de mama. Clínica e Instituição, 5, 59-70. 
Selltiz, C., Jahoda, M., Deutsch, M., \& Cook, S. M. (1972). Métodos de pesquisa nas relações sociais (I. O. Ribeiro, Trad.). São Paulo: Herder. (Obra original publicada em 1965)
Venâncio, J. L. (2004). Importância da atuação do psicólogo no tratamento de mulheres com câncer de mama. Revista Brasileira de Cancerologia, 50(1), 55-63.

Agradecimentos: Ana M. Lima, Amanda D. Dutra, Carolina R. Cardoso, Fernanda M. Massuco, Renata F. Ávila, Rita C. S. Mendes e Rita C. Gandini.

Natália Nogueira Teixeira de Menezes, psicóloga pela Universidade Federal de Uberlândia. Endereço para corespondência: Rua Isaac de Oliveira, 146, Bairro Progresso. Uberlândia-MG. CEP 38408-106. Telefones: (34) 32275759 e (34) 88638595. E-mail: nathie_ntm@hotmail.com

Vera Lucia Schulz, psicóloga pela Universidade Federal de Uberlândia. E-mail: vera_psico@hotmail.com. Rodrigo Sanches Peres, doutor em Psicologia pela Universidade de São Paulo, é professor do Programa de Pós-Graduação em Psicologia da Universidade Federal de Uberlândia. E-mail: rodrigosanchesperes@ yahoo.com.br 